\title{
LA EDUCACIÓN EN VALORES DE LOS ESTUDIANTES UNIVERSITARIOS: UNA EXPERIENCIA EN EL CENTRO UNIVERSITARIO MUNICIPAL DE JAGUEY GRANDE
}

\author{
Por Yanara Hernández Mato* \\ Centro Universitario Municipal Jagüey Grande, Matanzas Cuba \\ yanara.hernandez@umcc.cu \\ Raquel Pérez Cano** \\ Centro Universitario Municipal Jagüey Grande, Matanzas Cuba \\ raquel.perez@mcc.cu
}

Recibido: 29 /09/2016 Aceptado: 24/01/2017

\begin{abstract}
Resumen
La formación integral del estudiante universitario constituye el objetivo fundamental del proceso de formación del profesional en la educación superior cubana para ello se necesita una correcta labor educativa dirigida a la formación de valores humanos imprescindibles para un eficaz desempeño profesional y social. El presente trabajo demuestra que el Proyecto Educación y valores por una convivencia armónica contribuye a la formación de valores en los estudiantes universitarios, estudiantes de los restantes niveles de enseñanza y la comunidad, mediante una intensa labor educativa y político-ideológica en el Centro Universitario Municipal de Jagüey Grande, además potencia el
\end{abstract}

Master en Ciencias de la Educación y Especialista en Trabajo Social, aspirante en el Doctorado Curricular- Colaborativo en Ciencias Pedagógicas, profesora Auxiliar del Departamento de Ciencias Sociales del Centro Universitario Municipal de Jagüey Grande, Matanzas, Cuba.

** Master en Ciencias de la Educación Superior, profesora Auxiliar. Directora del Centro Universitario Municipal Jagüey Grande, Matanzas Cuba. 
perfeccionamiento moral de los estudiantes, que a su vez son parte activa de la comunidad, trabajan por, en y para ella.

Palabra Clave: Educación - Valores - Formación integral.

\section{Abstract}

The university student's integral formation constitutes the fundamental objective in the professional formation process in the Cuban higher education for it is necessary a correct educational work guided to the formation of human values indispensable for an effective professional and social performance. The present work demonstrates that the Project Education and Values for a Harmonic Coexistence contributes to the formation of values in the university students, students of the remaining teaching levels and the community, by means of an intense educational and political-ideological work in the Municipal University Center of Jagüey Grande, besides potency the moral perfecting of the students, that in turn are active part of the community, they work for, in and for it.

Keywords: Education - Values - Integral formation.

\section{Introducción}

La primera década del siglo XXI se caracterizó por una profunda crisis sistémica del régimen socioeconómico capitalista imperante a nivel mundial, crisis que se extendió más allá de lo económico manifestándose también en lo político, social, alimentario, energético y ambiental. Esta situación ha llegado hasta nuestros días y genera serios problemas de orden ético y axiológico que se expresan no solo a nivel de sociedades específicas sino que trascienden a escala universal.

En Cuba a pesar de los esfuerzos realizados para contrarrestar los efectos de la crisis de valores imperante en el mundo, se ha constatado la existencia de síntomas e indicadores que demuestran un significativo deterioro de valores humanos imprescindibles para el logro de una convivencia ordenada y desarrolladora. Varios investigadores han determinado los factores de carácter interno y externos que condicionan la crisis de valores en la sociedad cubana actual y plantean la necesidad de un accionar coherente, sistemático e integrado de los diferentes factores socializadores para educar en valores.

Los Centros Universitarios Municipales (CUM) surgidos como parte del proceso de Universalización de la Educación Superior, forman parte de la infraestructura de los centros de educación superior y tienen el propósito de garantizar el amplio acceso de la población, en su mayoría trabajadores, a los estudios universitarios. El trabajo docente-educativo que desarrollan estas instituciones tiene como principal prioridad lograr la correcta aplicación del 
enfoque integral para la labor educativa y el trabajo político-ideológico, dirigido a alcanzar una formación integral en los futuros egresados.

La observación sistemática del proceso docente-educativo en las distintas filiales universitarias del CUM por los profesores que imparten las diferentes asignaturas, las entrevistas efectuadas a los tutores, responsabilizados con la labor educativa personalizada, y el resultado de diferentes técnicas aplicadas a los estudiantes, ha evidenciado la existencia de un deterioro de determinados valores como la honestidad, la responsabilidad, la solidaridad, la honradez, la laboriosidad, así como, un cambio en la jerarquía de estos. A lo anterior se suma la falta de un accionar coherente, sistemático e integrado de los diferentes factores socializadores para educar en valores. El proceso de esa educación y de su formación en las jóvenes generaciones, como producto de la interiorización de aquellos contenidos valorados socialmente como positivos, está condicionado por el desarrollo y la experiencia histórica, social e individual de cada sujeto y en él influyen, entre otros factores: el medio familiar, la ideología imperante a nivel de la sociedad, la situación económico-social, la preparación educacional que se recibe en las instituciones formadoras y el entorno social donde interactúa el joven, básicamente el grupo del barrio y la comunidad. El problema científico de la investigación se define en los siguientes términos: ¿cómo contribuir a la educación en valores de los estudiantes del Centro Universitario Municipal Jagüey Grande?, para darle solución se crea el proyecto universitario Educación y valores por una convivencia armónica que tiene como objetivo contribuir a la formación de valores en los estudiantes universitarios, estudiantes de los restantes niveles de educación y la comunidad, mediante una intensa labor educativa y político-ideológica a partir de un accionar coherente, sistemático e integrado de los diferentes factores socializadores responsabilizados con la educación en valores. Se realizó un diagnóstico inicial para estudiantes y profesores, se imparten 3 cursos de postgrados sobre el tema con 188 cursistas, se han desarrollado clases metodológicas, instructivas y abiertas para demostrar cómo contribuir a la implementación del Programa de valores desde la instrucción, se impartió el curso de capacitación a los cuadros de las entidades del territorio, se desarrollaron talleres, conferencias y conversatorios sobre la vida y obra de José Martí, se imparten cursos de extensión en diferentes organismos, se celebra el 4to lunes de cada mes en la FUM el lunes martiano, todos los profesores propios de las FUM pasaron el curso. Superación política e ideológica para profesores universitarios, se realizan en cada centro matutinos teniendo en cuenta las efemérides locales, nacionales y los acontecimientos internacionales y para esto se creó una carpeta en soporte digital con materiales de apoyo como discursos de nuestros principales dirigentes, videos y bibliografía, se generalizan las investigaciones dirigidas a profundizar los conocimientos sobre temáticas diversas que contribuyan a la formación de valores y al fortalecimiento de la 
preparación político-ideológica.

El resultado de estas acciones se evidencia en la mejor preparación de estudiantes y profesores y sus logros han sido presentados en eventos como Humanística 2013 del ISP Juan Marinello, la Conferencia Científica Internacional de la UMCC y el evento provincial Universidad 2014. Actualmente se siguen implementando las acciones concebidas en función del perfeccionamiento moral de los estudiantes, que a su vez son parte activa de la comunidad, trabajan por, en y para ella.

\section{Desarrollo}

La formación integral del estudiante universitario constituye el objetivo fundamental del proceso de formación del profesional en la educación superior cubana, para ello es preciso encaminar el trabajo docente-educativo no solo a la adquisición de conocimientos, hábitos y habilidades para el ejercicio futuro de la profesión, también es necesaria una correcta labor educativa dirigida a la formación de valores humanos imprescindibles para un eficaz desempeño profesional y social. Las transformaciones a realizar en aras de lograr tal objetivo constituyen un reto de orden pedagógico y didáctico atendiendo a que requieren el diseño e implementación de un modelo interdisciplinario capaz de proyectar en toda su complejidad el desarrollo integral deseado.

La formación integral del estudiante universitario ha de transcurrir en el proceso educativo tanto en la esfera curricular como extracurricular. El proceso docente educativo tiene como objetivo central, formar integralmente al estudiante y prepararlo para que se desempeñe social y profesionalmente, la finalidad es preparar a los futuros profesionales en la adquisición de conocimientos y en la formación de habilidades y valores en correspondencia con el diseño del modo de actuación del modelo del profesional. Se desarrolla a través de diferentes formas organizativas que sitúan a los estudiantes como sujetos activos de su aprendizaje, jugando un papel rector la práctica laboral investigativa para permitir la vinculación del estudiante con el objeto de la profesión, coadyuvando de esta manera a la formación ética del futuro egresado.

Para lograr esto, las políticas y los modelos educativos requieren con urgencia estimular alternativas que potencien la evaluación humana, una de las vías es orientar el proceso formativo hacia al desarrollo de la personalidad de los estudiantes mediante su implicación activa y consciente en su propia formación.

Actualmente se realizan importantes esfuerzos para lograr una sólida formación humanística en los estudiantes, fundamentalmente mediante el fortalecimiento de la labor de formación axiológica y político-ideológica, sin embargo, las prácticas habituales que se realizan resultan insuficientes ante las exigencias que impone la contemporaneidad las que obligan cada vez más a la adquisición e implementación de un modelo interdisciplinario en el que los 
Revista de la Escuela de Ciencias de la Educación, año 13, nRo. 12, vol. 1, enero a Junio de 2017. Páginas 63-78. ISSN 1851-6297 - ISSN 2362-3349 (EN LINEA). LA EDUCACIÓN EN VALORES DE LOS ESTUDIANTES UNIVERSITARIOS: UNA EXPERIENCIA EN el centro universitario municipal de Jaguey Grande. Yanara Hernández Mato - Raquel Pérez Cano.

estudiantes desempeñen un papel activo en su propio proceso de formación. El sistema de educación en todos los niveles de enseñanza tiene la tarea suprema de formar hombres y mujeres capaces de llevar adelante las transformaciones económicas, políticas y sociales necesarias en cada momento histórico. Para ello, es preciso que el proceso educativo se centre no solo en lo instructivo, en la transmisión de conocimientos y la formación de habilidades, sino que se extienda más allá, al terreno afectivo, a partir de la comprensión del hombre como ser humano en el que se manifiesta una estrecha unidad de lo racional y lo emocional, elemento de imprescindible consideración para la formación de valores.

Autores de diversas latitudes se han referido a la educación en valores desde una concepción educativa que estimule el desarrollo cognoscitivo, afectivo y conductual de los escolares, a la vez que los motive, los interese, los incite a valorar, a dialogar y a participar en la toma de decisiones que lleven a un adecuado comportamiento: Montemayor (1996, España), Martínez (1998, Argentina), Jeréz (1998, México), Fabelo (2000, Cuba), González (2000, Cuba), Izquierdo (2000, Cuba), Ojalvo (2002, Cuba), Báxter (2003, Cuba), Arana (2005, Cuba); Batista (2001, Cuba).

Horruitiner P. define el término formación en la educación superior cubana como: "(...) el proceso sustantivo desarrollado en las universidades con el objetivo de preparar integralmente al estudiante en una carrera universitaria y abarca, tanto los estudios de pregrado como los de postgrado" (Horruitiner, 2006, p.17). El autor identifica tres dimensiones esenciales en el proceso de formación: instructiva, desarrolladora y educativa, las que en su integración posibilitan la formación de un profesional capaz de un desempeño social exitoso.

En el marco de la concepción filosófica asumida, la investigación se encuentra orientada a la educación en valores en el plano subjetivo. El valor se entiende, según Fabelo como: "componente de la estructura de la personalidad, que ejerce la función reguladora socialmente significativa de la conducta y las actitudes hacia el mundo circundante y sus relaciones con él. Constituye una guía general de la conducta que se deriva de la experiencia y le da sentido a la vida del individuo" (Fabelo, 1996, p. 50).

Se ha considerado, en este trabajo, tomar la educación en valores por su concepción de lograr la formación de una personalidad integral que tenga su base en los valores ya adquiridos desde sus relaciones sociales en el contexto en que se desarrolla, y la conversión de este aprendizaje en una proyección de modos de actuación positivos traducidos en componentes de la formación ciudadana. La Educación en Valores tiene como objetivo el alcance de una personalidad desarrollada o en desarrollo, la que se entiende, "al caracterizar a un individuo concreto donde el sistema de procesos y funciones que la forman se encuentran estructurados de manera armónica, en un proyecto de 
vida realista, donde predomina la autodirección consciente de los esfuerzos del individuo para lograr el desarrollo de sus potencialidades en forma creadora, así como su participación en la actividad social de acuerdo con valores de contenido progresista" (D’Angelo, 1996, p.4).

La educación en valores se expresa a través de la relación estudiante-profesor donde es precisamente la calidad de la relación, la que cambia el estilo de la comunicación con el estudiante y conduce a transformaciones en su crecimiento personal y valores. Ello implica enmarcar el proceso educativo dentro de límites siempre flexibles, en el que se respeten las diferencias y permita el establecimiento de una comunicación dialógica, ajena a todo autoritarismo o igualitarismo. La superación integral de los profesores constituye un elemento clave para el éxito en el desarrollo de la labor educativa, para ello es necesario que se sustente no solo en la transmisión de los conocimientos que se requieren sino que abarque un tratamiento certero a los componentes afectivo y conductual con el fin de lograr el convencimiento y una actitud responsable en el accionar cotidiano de cada profesor.

La formación del profesional supone no sólo brindar los conocimientos necesarios para el desempeño profesional, sino también tener en cuenta otros aspectos de igual relevancia. Se identifican tres dimensiones esenciales (Horruitiner, 2006):

- La dimensión instructiva relacionada con la idea de que para preparar un profesional resulta necesario instruirlo. Sin instrucción no hay formación posible. Al respecto Martí J. expresó:" Instrucción no es lo mismo que educación: aquella se refiere al pensamiento, y ésta principalmente a los sentimientos. Sin embargo, no hay buena educación sin instrucción. Las cualidades morales suben de precio cuando están realzadas por las cualidades inteligentes" (Martí, 1975, XIX, p. 375).

Martí J. enseñó lo necesario de desarrollar un pensamiento flexible, creativo, que conduzca a los estudiantes a pensar por sí mismos; en su combate al dogmatismo y a la aceptación de verdades absolutas y lo expresa al decir: "Y pensamos que no hay mejor sistema de educación que aquel que prepara al niño a aprender por sí. Asegúrese a cada hombre el ejercicio del sí propio" (Mena, Álvarez y Garlobo, 2004).

En el proceso de formación de profesionales se ha de tener en cuenta la participación del estudiante para lograr un aprendizaje sólido y al respecto Martí dice: "Deduzca por si las lecciones directas y armónicas que lo dejen enriquecido con sus datos a la vez que fortificado con el gusto de haberlas descubierto".

- La dimensión desarrolladora, su esencia es el vínculo entre el estudio y el trabajo, se requiere desarrollar en las competencias profesionales para asegurar su desempeño laboral exitoso. 
La obra martiana es pródiga en sus ideas acerca de la relación entre la teoría y la práctica, constituye una generalidad en su ideario pedagógico, concibe el proceso de enseñanza con clases donde predominara la práctica, el contacto con la naturaleza, con la realidad, con lo autóctono del país, y de su región, consideraba que solamente así se podía formar un hombre que respondiera a las necesidades de la sociedad y al respecto expresó: "las escuelas deben volverse del revés; del banco de sentarse debe hacerse banco de herrador o carpintero: del puntero de pizarra debe hacerse arado (Martí,1975, III, p. 314).

Es el vínculo entre el estudio y el trabajo, reconocido en el ideario pedagógico martiano, uno de los principios de la pedagogía cubana y elemento esencial en la formación de los profesionales, al respecto expresó también: "El hombre crece con el trabajo que sale de sus manos...el que debe su bienestar a su trabajo o ha ocupado su vida en crear y transformar fuerzas, y en emplear las propias, tiene el ojo alegre, la palabra pintoresca y profunda, la espaldas anchas, y la mano segura...esos que hacen al mundo...tienen cierto aire de gigantes dichosos, e inspiran ternura y respeto" (Martí, 1975, VIII, p. 285).

Insistió en su obra en la necesidad de eliminar el divorcio entre la teoría y la práctica; entre el estudio y el trabajo, y defendió la fusión de ellas en la obra educativa de la escuela cubana al decir que: "puesto que a vivir viene el hombre, la educación ha de prepararlo para vivir. En la escuela se ha de aprender el manejo de las fuerzas con que en la vida se ha de luchar. Escuelas no debía decirse, sino talleres. Y la pluma debía manejarse por la tarde en las escuelas; pero por la mañana, la azada" (Martí, 1975, XIII, p. 53).

- La dimensión educativa, elemento de primer orden en el proceso de formación, se encarga de la formación de la personalidad de los jóvenes, en particular en lo referido a aquellos valores que caracterizan su actuación profesional, y constituye la idea rectora principal y la estrategia más importante del proceso de formación.

Es de suma importancia el legado martiano acerca de la enseñanza orientada a la formación de elevados sentimientos y principios morales: la educación para el patriotismo. Insta en la educación de las virtudes humanas y reconoce como la primera virtud a la dignidad, es necesario crear hombres dignos, para lograr hombres justos, al respecto dice: "El hombre, que lleva lo permanente en sí, ha de cultivar lo permanente; o se degrada, y vuelve atrás, en lo que no lo cultive. A lo transitorio se esclavizan y venden los que no saben descubrir en sí lo superior y perdurable" (Martí, 1975, V, p. 284).

El proceso de formación debe encaminarse a lograr un profesional integral, que entre sus valores esenciales estén: patriotismo, responsabilidad, solidaridad, honestidad, honradez, dignidad, humanismo y justicia; que piense, sienta, valore, ame, se comporte y actúe en correspondencia con el momento en 
que le correspondió vivir. Significativo resulta el pensamiento pedagógico martiano, sus ideas sobre la adaptación de la escuela y de la educación a las necesidades de los individuos para que puedan marchar a la par de los tiempos y sobre ello expresó: "La educación tiene un deber ineludible para con el hombre, no cumplirlo es un crimen: conformarle a su tiempo sin desviarle de la grandiosa y final tendencia humana. Que el hombre viva en analogía con el universo, y con su época..." (Martí, 1961, p. 18).

El momento actual se caracteriza por la existencia de una profunda necesidad de transformar desde el punto de vista socioeconómico el territorio jagüeyense, proceso que requiere de una intensa transformación en el terreno ético, en la que se abandonen dogmas y estilos caducos, pero se preserve lo más valioso de la moral revolucionaria nacional y local edificada a lo largo de la historia. Las instituciones educativas tienen en este proceso un importante papel, enseñar a pensar a los estudiantes, a entender la totalidad social en que se encuentran insertados, a valorar por sí mismos y a identificar e interpretar correctamente los elementos que forman parte de la cara oculta del capitalismo, lo expresado resulta esencial para promover cambios en las aspiraciones, motivaciones y conductas de los jóvenes del territorio.

Teniendo en cuenta las dificultades detectadas el proyecto pretende el logro de un clima educativo favorecedor de la formación axiológica en el territorio, a partir de un accionar coherente, sistemático e integrado de los diferentes factores socializadores responsabilizados con la educación de valores. La labor educativa es asumida como un momento de creatividad, sensibilidad e imaginación en la búsqueda de formas novedosas que hagan posible la formación y desarrollo de valores imprescindibles para una convivencia armónica y ordenada, que posibilite la realización de las transformaciones de carácter económico, político y social a desarrollar, en un ambiente constructivo y de colaboración.

En el Seminario Nacional de Preparación del Curso 2011-2012 del Ministerio de Educación Superior (MES) se plantearon los lineamientos que regirán el trabajo político-ideológico en las universidades donde se prioriza desarrollar valores éticos en los estudiantes, de ahí se desprende que el área de resultado clave 1 sea: profesional competente comprometido con la Revolución e incluya la formación integral, formación de valores y Educación Patriótico Militar, la enseñanza del Marxismo-Leninismo y la Historia de Cuba, los turnos de Debates Históricos Contemporáneos, una cultura medioambiental y de paz, el tratamiento al léxico y expresión oral (MES, 2011, p.9). Lo expresado exige el desarrollo de un intenso proceso de formación de valores en los planos curricular y extracurricular, a partir del establecimiento de un estrecho vínculo entre todas las disciplinas y asignaturas previstas en los planes de estudio de cada carrera, donde se logre una 
adecuada integración con la actividad investigativa, laboral, extensionista, y las tareas de impacto social en las que participan los estudiantes.

Las condiciones en que se desarrolla el proceso de formación del profesional en las filiales universitarias municipales en el municipio Jagüey Grande, así como en los niveles precedentes de enseñanza, exigen de un intenso trabajo educativo y político-ideológico encaminado a la formación de valores en los estudiantes. Las insuficiencias existentes en relación con el desarrollo de esta labor, constatadas en diversas investigaciones realizadas y en la práctica docente, muestran la necesidad de un accionar coherente e integrado sobre bases científicas, que conduzca a la incorporación de valores a la personalidad. Los objetivos y acciones concebidos en el proyecto tienen el propósito de encontrar solución a las necesidades educativas existentes y lograr una transformación positiva de la personalidad de los estudiantes en correspondencia con las exigencias que impone el entorno social. En correspondencia con lo expresado se considera que los objetivos y acciones planificadas responden a las necesidades existentes, por lo que el proyecto resulta pertinente.

El proceso de formación debe encaminarse a lograr un profesional integral, que entre sus valores esenciales estén: patriotismo, responsabilidad, solidaridad, honestidad, honradez, dignidad, humanismo y justicia; que piense, sienta, valore, ame, se comporte y actúe en correspondencia con el momento en que le correspondió vivir, significativo resulta el pensamiento pedagógico martiano, sus ideas sobre la adaptación de la escuela y de la educación a las necesidades de los individuos para que puedan marchar a la par de los tiempos y sobre ello expresó: "La educación tiene un deber ineludible para con el hombre, no cumplirlo es un crimen: conformarle a su tiempo sin desviarle de la grandiosa y final tendencia humana. Que el hombre viva en analogía con el universo, y con su época..." (Martí, 1961, p.18).

Martí en su obra reclamó la necesidad de la formación de elevados sentimientos y principios morales, la educación para el patriotismo, educar en la virtud, que los conocimientos que el hombre adquiera le permitan superarse a sí mismo, crear las vías de su autoperfeccionamiento moral y así lograr la fraternidad humana, al respecto escribió: "Es bella la fraternidad humana: es conmovedora, es pura, es necesaria: la simpatía es su forma, la unión su resultado, la grandeza común su espléndida creación" (Martí, I, p. 448).

El proyecto Educación y valores por una convivencia armónica tiene como objetivo general: Contribuir a la formación de valores en los estudiantes universitarios, estudiantes de los restantes niveles de enseñanza y la comunidad, mediante una intensa labor educativa y político-ideológica. 


\section{Objetivos específicos}

- Asegurar el desarrollo de acciones encaminadas a la realización del diagnóstico de las necesidades educativas existentes.

- Garantizar la realización de acciones dirigidas al fortalecimiento de la labor educativa y político-ideológica por la vía curricular en los centros de enseñanza del municipio.

- Potenciar el desarrollo de actividades de postgrado orientadas a la superación de los docentes en el orden educativo y político-ideológico.

- Garantizar la ejecución de actividades de extensión universitaria y de carácter sociopolítico que contribuyan a la formación de valores, tanto de los estudiantes implicados en el proceso formativo como de la comunidad en sentido general.

- Potenciar la realización de acciones dirigidas al fortalecimiento de la preparación cívica, jurídica, económica y medioambiental de la comunidad universitaria, en los restantes niveles de enseñanza y la comunidad.

- Propiciar el desarrollo de la investigación científica dirigida a una mayor profundización en el abordaje de temas vinculados al trabajo educativo y político-ideológico, la formación cívica, jurídica, económica y medioambiental.

- Garantizar el control de la implementación de las acciones concebidas en el proyecto.

Para el logro de los objetivos concebidos en el proyecto la metodología a seguir se centra en el desarrollo de: actividades docentes, actividades metodológicas, controles a las actividades docentes y metodológicas y, la estrecha vinculación entre las actividades de investigación, postgrado y extensión en aras de solucionar las limitaciones existentes. Se considera como un elemento de importancia vital la transformación no solo de la institución educativa, sino del medio socio-familiar y comunitario.

\section{Entidades participantes}

Filial Universitaria Municipal MES Jagüey Grande.

Filial Universitaria Municipal Pedagógica Jagüey Grande.

Filial Universitaria Municipal de Cultura Física Jagüey Grande.

Filial Universitaria Municipal de Salud Jagüey Grande.

Dirección Municipal de Educación Jagüey Grande.

Dirección Municipal de Cultura Jagüey Grande.

Dirección Municipal de Salud Jagüey Grande.

Dirección Municipal del INDER Jagüey Grande.

Dirección del CITMA en Jagüey Grande.

Emisora Radio Victoria de Girón Jagüey Grande.

Dirección de Trabajo Social Jagüey Grande. 


\section{Acciones realizadas:}

- Diagnóstico del nivel de desarrollo de los valores presentes en los estudiantes universitarios que estudian en el municipio y en los estudiantes de los restantes niveles de enseñanza. La población está constituida por 999 estudiantes del CUM Jagüey Grande distribuidos en las 4 filiales universitarias de la siguiente forma: MES (590), Medicina (229), Pedagógica (104), Cultura Física (76). Se tomaron como muestra 560 de ellos, lo que representa el $56 \%$ de los estudiantes universitarios del municipio.

- Diagnóstico del estado en que se encuentra la preparación políticoideológica de docentes y estudiantes del territorio en correspondencia con las necesidades.

- Diagnóstico de forma sistemática la evolución que experimentan los estudiantes como resultado de las acciones desarrolladas a través del proyecto.

- Impartir el posgrado: Superación política e ideológica para profesores universitarios.

- Impartir el posgrado: La educación en valores a través de la instrucción.

- Impartir el posgrado: La Educación en valores de la sociedad cubana actual.

- Desarrollar clases metodológicas, instructivas y abiertas para demostrar cómo contribuir a la implementación del Programa de valores desde la instrucción.

- Instrumentar a través de todas las asignaturas y disciplinas acciones encaminadas al fortalecimiento del dominio de la Historia de Cuba por los estudiantes.

- Mantener los turnos de Debate Histórico-Contemporáneo sobre elementos de carácter histórico y actual que contribuyan a la formación y desarrollo de valores y al logro de una preparación político-ideológica acorde con las necesidades de la construcción del socialismo en Cuba.

- Impartir el curso de capacitación a los cuadros de las entidades del territorio.

D Desarrollar talleres, conferencias y conversatorios sobre la vida y obra de José Martí.

- Efectuar conversatorios con figuras destacadas del territorio en la ciencia, la técnica, el deporte, la pedagogía, la defensa de la patria, la cultura, la historia u otras actividades de valía para la sociedad.

Realizar actividades en conmemoración a acontecimientos de carácter económico, político, social, medioambiental, jurídico, entre otros, asociados a la historia universal, nacional y local.

Asesorar el desarrollo de investigaciones encaminadas a la educación de valores éticos, estéticos y medioambientales a través de la programación radial de la emisora municipal Radio Victoria de Girón, por especialistas en 
el tema pertenecientes a la educación superior.

- Realizar trabajos de investigación de carácter teórico dirigidos a profundizar los conocimientos sobre temáticas diversas que contribuyan a la formación de valores y al fortalecimiento de la preparación político-ideológica, con énfasis en el desarrollo de investigaciones históricas.

- Analizar en el grupo gestor del proyecto los resultados del diagnóstico en sus diferentes etapas y el estado de cumplimiento de las acciones a realizar, con énfasis en las medidas a adoptar para solucionar las insuficiencias detectadas.

- Comunicación de los resultados parciales obtenidos al organismo que auspicia el proyecto.

- Evaluar el conocimiento sobre el Programa Director para la educación en el sistema de valores de la Revolución Cubana.

- Realizar en cada centro un taller sobre el Programa director para la educación en el sistema de valores de la Revolución Cubana.

- Implementar en cada centro la realización de matutinos teniendo en cuenta las efemérides locales, nacionales y los acontecimientos internacionales.

- Concebir una actividad que demuestre cómo desde tu centro de trabajo puedes influir en el fortalecimiento de los valores de la comunidad.

Estas acciones propician el desarrollo de una educación moral y valorativa en las aulas universitarias que tiene como finalidad el avance hacia un proceso educativo que va más allá de la mera asimilación de conocimientos y manejo de la información y se centra esencialmente en el logro de la unidad entre instrucción y educación con vistas a alcanzar una formación integral de los estudiantes. En este proceso el profesor juega un papel fundamental al convertirse en mediador entre un acervo cultural en constante transformación y la personalidad en formación de sus estudiantes, donde es necesario el establecimiento un clima acogedor que propicie el desarrollo de la investigación y la creatividad.

La educación en valores se expresa a través de la relación estudiante-profesor donde es precisamente la calidad de la relación, la que cambia el estilo de la comunicación con el estudiante y conduce a transformaciones en su crecimiento personal y valores. Ello implica enmarcar el proceso educativo dentro de límites siempre flexibles, en el que se respeten las diferencias y permita el establecimiento de una comunicación dialógica, ajena a todo autoritarismo o igualitarismo. La superación integral de los profesores constituye un elemento clave para el éxito en el desarrollo de la labor educativa, para ello es necesario que se sustente no solo en la transmisión de los conocimientos que se requieren sino que abarque un tratamiento certero a los componentes afectivo y conductual con el fin de lograr el convencimiento y una actitud responsable en el accionar cotidiano de cada profesor. 
El proyecto universitario jagüeyense ha comenzado a dar sus frutos. Esto se evidencia:

- El $100 \%$ de las personas responsables del proyecto en sus instituciones ha recibido la capacitación necesaria para accionar desde su centro en la labor educativa.

- El $100 \%$ de las entidades que participan en el proyecto diagnostica las principales necesidades educativas existentes.

- Se logra el desarrollo de la labor educativa y político-ideológica de manera consciente y científica en las actividades docentes que se realizan en el territorio en los diferentes niveles de educación, lo que produce una transformación significativa en los resultados obtenidos a través de diferentes indicadores

Se han desarrollado clases metodológicas, instructivas y abiertas para demostrar cómo contribuir a la implementación del Programa de valores desde la instrucción.

- Los docentes realizan acciones para la elevación de los conocimientos de Historia de Cuba a través de sus clases.

- El $100 \%$ de los estudiantes participa en los debates históricos y contemporáneos que se desarrollan en su centro de educación.

- El $100 \%$ de los docentes se supera para desarrollar el trabajo educativo y político ideológico a través de la actividad que realiza en cada centro de educación.

- Se supera política e ideológicamente el $100 \%$ de los cuadros y profesionales de las instituciones que participan en el proyecto.

- El $75 \%$ de los profesionales, cuadros y dirigentes del territorio participa en al menos un curso de postgrado encaminado al fortalecimiento de su preparación político-ideológica.

- El $100 \%$ de los estudiantes de los diferentes niveles de educación, participa activamente en las actividades de las cátedras honoríficas, la cátedra martiana, en conversatorios con figuras destacadas del territorio, actividades de conmemoración a acontecimientos históricos y en los proyectos socioculturales y comunitarios diseñados en función del desarrollo local.

- En el $100 \%$ de los proyectos socioculturales, comunitarios y de investigación se tienen en cuenta los elementos de carácter cívico, económico, medioambiental y jurídico necesarios.

Se presentó un trabajo en Humanística 2013 del ISP Juan Marinello, en la Conferencia Científica Internacional de la UMCC y el evento provincial Universidad 2014.

- Se continúa impartiendo el posgrado: La educación en valores a través de la instrucción con énfasis en el sector educacional.

Se celebra el 4to lunes de cada mes en la FUM el lunes martiano. 
- Todos los profesores propios de las distintas filiales universitarias del CUM pasaron el curso: Superación política e ideológica para profesores universitarios sobre el pensamiento martiano.

- Se generalizan las investigaciones dirigidos a profundizar los conocimientos sobre temáticas diversas que contribuyan a la formación de valores y al fortalecimiento de la preparación político-ideológica, con énfasis en el desarrollo de investigaciones históricas.

- Se realizan en cada centro matutinos teniendo en cuenta las efemérides locales, nacionales y los acontecimientos internacionales. Para lo cual se creó una carpeta en soporte digital con materiales de apoyo como discursos de nuestros principales dirigentes, videos y bibliografía.

El resultado científico que presenta la investigación es una estrategia educativa para contribuir a la educación en valores de los estudiantes del CUM de Jagüey Grande. Este resultado adquiere una significación teórica, dada en la sistematización de los referentes axiológicos, pedagógicos y didácticos que permitieron diseñar un sistema de actividades que conforman la estrategia educativa para contribuir a educar en valores a estudiantes y profesores del Jagüey Grande.

Asimismo la connotación práctica la determina, el hecho de contar con una herramienta de trabajo para el CUM que posibilita la vinculación y tratamiento de las actividades que en ella aparecen con las diferentes asignaturas del plan de estudio, proporcionando la interdisciplinariedad y el objetivo de contribuir a la formación ciudadana, política y moral del individuo. También permite darle salida al programa de Lengua Materna, Historia de Cuba, Debate Histórico-Contemporáneo y a los contenidos principales relacionados con la educación patriótica militar e internacionalista, jurídica, laboral económica, estética, ética y ambiental. Posibilita la transformación de la conducta hacia la comunidad y sus individuos y la suya propia, para cambiar lo que debe ser cambiado, en concordancia con su labor como miembro de nuestro municipio.

Se hace necesario en estos momentos erradicar la improvisación en el trabajo educativo y político-ideológico, gestionarlo a ciclo completo: planificación, organización, ejecución y control, para poder cumplir los objetivos previamente trazados después de realizados los diagnósticos correspondientes e incorporar la sistematicidad en el trabajo y sobre todo en el control para garantizar la eficacia que requiere el proceso de formación de valores.

\section{Conclusiones}

- Constituye una necesidad que en la sociedad cubana se formen jóvenes comprometidos con su historia y su país, para dirigir pedagógicamente la educación en valores es necesario sistematizar un cuerpo de conocimientos, criterios y reflexiones que sirvan de plataforma teórica general. 
La implementación del Proyecto universitario Educación y valores por una convivencia armónica se valora como una vía efectiva para la educación en valores de los estudiantes y profesores del municipio pues al comenzar su aplicación se puede inferir que: cuando los estudiantes y profesores conocen con precisión y claridad los elementos cognoscitivos y normas de conducta propias de los valores, a través de actividades que le permitan reflexionar, debatir y realizar valoraciones, están en mejores condiciones para sentirlos, tener voluntad para asumirlos como suyos y manifestarlos con frecuencia en su actuación cotidiana.

El proyecto Educación y valores por una convivencia armónica contribuye al perfeccionamiento moral de los estudiantes y profesores, que a su vez son parte activa de la comunidad, trabajan por, en y para ella, que en este caso se refiere al municipio Jagüey Grande.

\section{Referencias Bibliográficas}

- Acosta, H. (2007). Moral, ética y justicia. Revista Cuba Socialista. La Habana. Editorial del Comité Central del Partido Comunista de Cuba. No.43.

- Aldea, E. (2012). Estrategias educativas para trabajar en valores. --[en línea]. -- htpp// www.campusoci. org/fromvalores/htm. -- [Consultado: 26 DICIEMBRE. 2012]

- Bargh, J. A. (2003). Personality \& social change: attitude and social formation in a student community. [en línea]. [consultado: 8 diciembre 2012] Disponible en: http://www. google.com/scholar?hl=en\&q=related:iltwsm6r2nkj:scholar.google.com .

- Barriga, A. D. (2006). La educación en valores: avatares del currículo formal, oculto y los temas transversales. [en línea]. [consultado: 2 marzo 2008]. Disponible en: http:/www. um.es/ead/red/9/valores.pdf.

- Baxter, E. (2006). Diversidad de métodos para educar y evaluar lo logrado en la educación en valores. En: MINED. VII Seminario Nacional para Educadores. La Habana: Editorial Pueblo y Educación.

- Brady, L. (2011). Teacher Values and Relationship: Factors in Values Education. Australian Journal of Teacher Education: Vol. 36: Iss. 2, Article 5. Available at: http://ro.ecu.edu. au/ajte/vol36/iss2/5.

- Castellanos, A. V. (2003). Estrategia docente para contribuir a la educación de valores en estudiantes universitarios: su concepción e instrumentación en el proceso docente. Revista Pedagogía Universitaria. La Habana, CEPES, Vol.8, № 1.

- D' Angelo, O. (2005). Proyecto de vida y desarrollo personal. En Colectivo de autores. Pensando en la personalidad. 1ra ed. La Habana: Editorial Félix Varela.

- Domínguez, L. (2005). Identidad, valores y proyecto de vida. En Colectivo de autores. Pensando en la personalidad. 1ra ed. La Habana: Editorial Félix Varela.

- Fabelo, J. R. (2003). Los valores y sus desafíos actuales. 2da ed. La Habana: Editorial José Martí.

- Ginoris, O.; Addine, F.; Turcaz, J. (2006). Didáctica general. [CD-ROM]. Maestría en Ciencias de la Educación Superior. CEDE .UMCC. 
- González, V.; Kraftchenko, O.; Ojalvo, V. (2003). El diagnóstico psicopedagógico de los valores. Revista Pedagogía Universitaria. La Habana, CEPES, Vol.8, No.1.

- Horruitinier, P. (2006). La universidad cubana. El modelo de formación. La Habana: Editorial Félix Varela.

- Martí, J. (1993). Obras escogidas. Tomos I, II y III. La Habana: Editorial Ciencias Sociales.

- Mendoza, L. (2003). Axiología y cultura en José Martí.

- Ojalvo, V. (2003). Educación de valores. Revista Pedagogía Universitaria. La Habana, CEPES, Vol.8, No.1.

- PCC Programa director para la educación en el sistema de valores de la Revolución Cubana. La Habana: Editora Política. 2012.

- Ramos, G. /sa/. La dimensión axiológica de la formación profesional universitaria: un reto frente a la globalización neoliberal. [en línea] [Consultado: 22 julio 2007]. Disponible en: http://intraweb.umcc.cu/cede/aeses.htm

- Romero, C. (2007). La formación axiológica en la universidad: una experiencia cubana. [CD-ROM]. Universidad 2008. CEDE. UMCC. 